baseline variables. Each patient was followed at each clinic visit for 12 months for development of a physician-reported irAE.

For irAE, site and grade were recorded as documented by the provider. At diagnosis and each follow-up visit, we collected: 1) adjustments to ICI, immunosuppression, and hormone therapy.

Continuous variables were summarized using mean and standard deviation (SD) or median and interquartile range (IQR). Categorical variables were summarized using frequencies and percentages. Time to development and resolution of irAE were calculated using Kaplan-Meier curves. RESULTS/ANTICIPATED RESULTS: Among 131 patients, two-thirds were men, and 60\% were Caucasian with a mean age was 65 years. Nearly $40 \%$ had an Eastern Cooperative Oncology Group performance score of 2 or higher. A small proportion $(3.1 \%)$ had an autoimmune disorder. Nearly half had lung cancer $(49.6 \%)$, and several had received radiation (33.6\%). Over $70 \%$ were former or current smokers. In total, 57 patients (43.5\%) developed an irAE, resultng in a total of 95 irAE, at a median of 208 days (126 days, not reached). The most common irAE included dermatitis, thyroiditis, pneumonitis, and hepatitis. Of $95 \mathrm{irAE}$, half were grade $1,30 \%$ were grade 2 , and nearly $20 \%$ were grade 3 or higher. Median time to resolution was 85 days (56-183 days). DISCUSSION/SIGNIFICANCE OF FINDINGS: This study demonstrates that irAE are clinically impactful in this relatively unfit, medically complex population, which may be a more accurate reflection of patients receiving ICI in everyday practice. This study showcases that this population is susceptible to irAE, although this needs to be examined in larger, prospective trials.

55715

\section{Quantification of Neonatal THC Exposure Following Prenatal Marijuana Use}

Stefanie Kennon-McGill, Ph.D., Heather Moody, Jeff Moran, Ph.D., and Laura James, MD

University of Arkansas for Medical Sciences

ABSTRACT IMPACT: Quantification of neonatal THC exposure will allow for better insight into how THC exposure correlates with neurodevelopmental outcomes. OBJECTIVES/GOALS: Tetrahydrocannabinol (THC) use has become increasingly prevalent in recent years, including among pregnant women. However, few, if any, clinical studies have quantified precise in utero exposure levels of THC during pregnancy. Our study aims to fill this gap by using analytical methods to quantify THC in mother and baby following prenatal THC use. METHODS/STUDY POPULATION: Pregnant women were asked to give a self-report of all cannabis and cannabinoid use during pregnancy, including dose, frequency, and route of consumption. Upon arrival at the labor and delivery unit, maternal blood samples were collected. Immediately following birth and 24 hours after birth, umbilical cord and neonatal blood samples were collected, respectively. All blood samples were analyzed using tandem liquid chromatography-mass spectrometry (LC-MS) for the presence of THC, tetrahydrocannabinol carboxylic acid (THC$\mathrm{COOH})$, and hydroxy-tetrahydrocannabinol (THC-OH). Maternal THC and metabolite levels were compared to both cord and neonate samples. RESULTS/ANTICIPATED RESULTS: To date, we have collected 3 mother-infant sample dyads and 4 mother-infant control samples. We anticipate collecting a total of 20 mother-infant samples from each group. We will quantify levels of THC and its metabolites in maternal samples and compare these to cord and infant samples.
We expect that THC/metabolite levels will vary as a function of dose and frequency of consumption. We also expect that THC/metabolites will be higher in umbilical cord blood relative to neonatal blood. DISCUSSION/SIGNIFICANCE OF FINDINGS: This study is among the first to directly measure exposure in the neonate following prenatal cannabis use. Quantification of THC/metabolite concentrations will be supplemented with developmental evaluations of infants at 6 and 12 months of age in order to gain better insight into how THC exposure correlates with neurodevelopmental outcomes.

73662

Racial Disparities in Septic Shock Mortality: Results from the OneFlorida Data Trust Cohort

Lauren Page Black, MD, MPH, University of Florida College of Medicine - Jacksonville; Charlotte Hopson, MS, University of Florida College of Medicine - Jacksonville, Center for Data

Solutions; Elizabeth DeVos, MD, MPH, University of Florida College of Medicine - Jacksonville; Michael Puskarich, MD, MS, University of Minnesota; Rosemarie Fernandez, MD, University of Florida College of Medicine; Faheem Guirgis, MD, University of Florida College of Medicine - Jacksonville; Cynthia Garvan, PhD, University of Florida College of Medicine

ABSTRACT IMPACT: Identifying racial disparities in septic shock mortality, a common and lethal condition, can inform future research and policy efforts aimed at understanding the drivers these disparities and addressing the underlying factors in order to reduce disparities and improve health. OBJECTIVES/GOALS: Septic shock is a major public health problem with significant mortality. Existing data indicate racial disparities in sepsis incidence, but evidence is limited on differences in septic shock outcomes. Our objective was to determine the association between race and septic shock mortality in a statewide cohort while controlling for clinical factors. METHODS/STUDY POPULATION: This was a retrospective analysis of septic shock patients in the One Florida Data Trust between 2012-18. Data was collected regarding age, sex, race, insurance status, and selected comorbid conditions [liver disease, hypertension, chronic obstructive pulmonary disease (COPD), congestive heart failure $(\mathrm{CHF})$, end-stage renal disease (ESRD), and human immunodeficiency virus infection (HIV)]. To account for severity of illness, we assigned Sequential Organ Failure Assessment scores for components based on laboratory values (labSOFA), and collected data on mechanical ventilation use and initial lactate.

The primary outcome was 90-day mortality. The Least Absolute Shrinkage and Selection Operator (LASSO) method was used for variable selection for the multivariable regression model. RESULTS/ANTICIPATED RESULTS: There were 13,932 septic shock patients with a mean (SD) age of 61(16) years. Of these, $68 \%$ identified as white, $28 \%$ as black, $2.1 \%$ as Hispanic, and $2.0 \%$ as other races. 90-day mortality was $32 \%(n=4,437)$ and $59 \%$ required mechanical ventilation. Significant independent predictors of mortality in the regression model were age (OR 1.04; $\mathrm{p}<0.01$ ), black race $(1.72 ; \mathrm{p}<0.01)$, lactate $(1.10 ; \mathrm{p}<0.01)$, mechanical ventilation $(3.62 ; \mathrm{p}<0.01)$, labSOFA $(1.18 ; \mathrm{p}<0.01)$, history of liver disease $(1.75 ; \mathrm{p}<0.01)$, hypertension $(0.70 ; \mathrm{p}<0.01)$, COPD $(0.87 ; \mathrm{p}<0.01)$, $\operatorname{CHF}(1.18 ; \mathrm{p}<0.01), \operatorname{HIV}(1.30 ; \mathrm{p}=0.05)$, and the interaction between age and black race. Black patients had 1.72 times the odds of mortality compared to white patients. For every one-year decrease in age, 
black patients had a $1 \%$ increased odds of mortality (OR 1.01; $\mathrm{p}<0.01$ ). DISCUSSION/SIGNIFICANCE OF FINDINGS: Black patients have increased odds of dying from septic shock compared to white patients after controlling for age, selected comorbid conditions, and markers of illness severity. Future work is needed to move beyond demonstrating septic shock disparities and towards understanding the underlying factors.

\section{1}

Current implementation of expedited partner therapy for the treatment of $\mathbf{N}$. Gonorrhoeae and C. Trachomatis infection: Integrating mixed methods with costeffectiveness analysis

Emily Ann Groene Faherty ${ }^{1}$, Kumi Smith ${ }^{2}$, Christy M. Boraas ${ }^{3}$, Sarah M. Lofgren ${ }^{3}$ and Eva A. Enns ${ }^{2}$

${ }^{1}$ University of Minnesota Clinical and Translational Science Institute; ${ }^{2}$ University of Minnesota School of Public Health; ${ }^{3}$ University of Minnesota Medical School

ABSTRACT IMPACT: This work will estimate current EPT implementation in Minnesota and provide cost-effectiveness analyses of different implementation scenarios to inform STI treatment policy. OBJECTIVES/GOALS: This research aims to 1) assess current implementation of Expedited Partner Therapy (EPT) as treatment for C. Trachomatis (chlamydia) and N. Gonorrhoeae (gonorrhea) among healthcare providers in Minnesota and to 2) simulate the current burden of chlamydia and gonorrhea infections to test the costeffectiveness of increasing EPT implementation. METHODS/ STUDY POPULATION: We will conduct key informant interviews (KII) and an online survey of health providers across the continuum of care for chlamydia and gonorrhea treatment. Based on experience in prior studies, the KII sample size is expected to be about 15 informants. KIIs will be carried out among providers who submitted EPT protocols to the Minnesota Department of Health to understand how EPT is currently being implemented. KII results will inform the online survey of health providers, which will estimate how many providers across the state provide EPT. We will distribute the survey through Minnesota health provider networks to achieve a sample of at least 500 health providers. The KII and survey results will inform model structure and parameter values for a compartmental cost-effectiveness model of EPT. RESULTS/ANTICIPATED RESULTS: Initial results from KII pilots suggest that EPT is primarily provided through a paper script for the sexual partner of a patient who tests positive for CT or NG by the treating provider. Less commonly, a patient's partner who is already a patient in the health system may receive notification and treatment through the provider. While EPT is legal in Minnesota, concerns about medical liability for adverse reactions and difficulty obtaining paper scripts in electronic workflows are barriers to implementation. The statewide survey will include questions to estimate the likelihood of EPT provision among providers when these concerns are addressed. These figures will be integrated into the cost-effectiveness model to simulate outcomes and costs across different EPT implementation scenarios. DISCUSSION/SIGNIFICANCE OF FINDINGS: The statewide survey will define cost-effectiveness model parameters, including the proportion of providers in the state who currently provide EPT or would be willing to provide EPT under different scenarios. Study findings will be shared with health provider networks and health departments to inform STI treatment procedures and state EPT policies.

\section{Clinical Trial}

27646

Spinal Control Impairments During Two Clinical Tests of Lower Limb Movement in People with and without Low Back Pain

Stacey Chen, Quenten Hooker, Vanessa Lanier, Linda Van Dillen Washington University of St. Louis

ABSTRACT IMPACT: Our work may be able to impact the examination and clinical decision making of a clinician to identify and target movement impairments to treat people with low back pain. OBJECTIVES/GOALS: Test if the magnitude of spinal control impairments is different in two clinical tests of lower limb movement in people with and without low back pain (LBP). The impairment is defined as the time difference between the start of limb to lumbopelvic motion. Also, test if the magnitude of impairments across tests is associated with LBP intensity and function. METHODS/STUDY POPULATION: 18 controls and 21 people with LBP $(51.6 \% \mathrm{~F}$, $34.5 \pm 11.5 \mathrm{yrs})$ participated in a cross-sectional, laboratory-based study. Subjects completed the modified Oswestry Disability Questionnaire (LBP-related functional limitation measure; 0$100 \%$ ) and the Numeric Pain Rating Scale (LBP intensity; average pain prior 7 days; 0 -10) self-report surveys and clinical tests of hip medial and lateral rotation performed in prone. A three-dimensional motion capture system was used to capture angular lumbopelvic and hip motion across time. A 2x2 mixed model ANOVA will be used to examine the effects of group, hip rotation test, and group $\mathrm{x}$ hip rotation test. Separate bivariate correlations will be used to quantify the association of magnitude of the impairment to (1) average LBP intensity and (2) LBP-related functional limitation. RESULTS/ANTICIPATED RESULTS: We hypothesize that, compared to healthy controls, people with LBP will display a greater magnitude of impairment across the hip medial and lateral rotation tests. In addition, we hypothesize that the magnitude of the difference in impairment between people with LBP vs controls will be larger during the hip lateral rotation test compared to the hip medial rotation test. Finally, we hypothesize that in people with LBP the magnitude of the impairments across tests will be associated with LBP intensity and LBP-related functional limitation. DISCUSSION/ SIGNIFICANCE OF FINDINGS: If our hypotheses are supported, the hip rotation tests would further be recognized as a key part of a clinician's examination and an important target for treatment of LBP.

35991

\section{Towards a Novel Robotic Control Scheme to Improve} Lower Extremity Movement Post-Stroke

Tom Ruopp, Dr. Brian Schmit and Dr. Sheila Schindler-Ivens Marquette University

ABSTRACT IMPACT: Effective robotic pedaling therapy would allow stroke survivors a precision, customized, and adaptable therapy to help recover lower extremity function. OBJECTIVES/ GOALS: It has been observed that people post-stroke can pedal each limb individually but not simultaneously when the bicycle is splitcrank. This implies that lower extremity movement difficulties are affected more by interlimb rather than unilateral coordination deficits. This work seeks to further develop a robotic split-crank 